\title{
Analysis Methodologies and Experimental Benchmarks for Eddy Current Testing
}

\author{
E. Cardelli ${ }^{1}$, Senior Member, IEEE, A. Faba ${ }^{1}$, R. Specogna ${ }^{2}$, A. Tamburrino ${ }^{3}$, F. Trevisan ${ }^{2}$, and S. Ventre ${ }^{3}$ \\ ${ }^{1}$ Dip. di Ingegneria Industriale, University of Perugia, Perugia 06124, Italy \\ ${ }^{2}$ Dip. di Ingegneria Elettrica, Gestionale e Meccanica, 33100 Udine, Italy \\ ${ }^{3}$ DAEIMI, 43-Cassino (FR) I-03043, Italy
}

\begin{abstract}
In this paper, we present numerical models and experimental benchmarks for eddy current testing (ECT) applications, developed within the methods and applications of nondestructive electromagnetic diagnostic (MADEND) research project. We compare and validate two different numerical methods for eddy current calculation in the framework of ECT (an integral formulation implemented on a parallel computer system and a discrete formulation based on a geometric approach) by using specifically designed experimental benchmarks and a commercial finite element method based on a differential formulation.
\end{abstract}

Index Terms-Discrete geometric approach, eddy current testing (ECT), integral formulation.

\section{INTRODUCTION}

$\mathbf{T}$ HE aim of this work is to present some analysis methodologies and experimental benchmarks specifically developed for the solution and validation of the direct problem in eddy current testing (ECT). These research activities have been developed in the framework of the Italian research project methods and applications of nondestructive electromagnetic diagnostic (MADEND). Of the various analysis methods, the integral formulation implemented in the CARIDDI code has been greatly improved thanks to a parallel implementation, and to a reduction of the computational effort from $O\left(n^{3}\right)$ to $O(n \log (n))$ achieved by using the precorrected fast Fourier transform (FFT) method and the fast multipole method (FMM) [1]. In addition, a discrete geometric formulation has been developed based on a geometric reinterpretation of the physical laws and of the constitutive laws of magneto-quasistatics referred to a pair of cell complexes, one being the dual of the other [2]. Typical output quantities in both approaches can be the variations, produced by a defect, in the magnetic flux density (at prescribed points) or in the impedance at coil leads. In order to validate and compare these formulations, a number of experimental benchmarks [3] have been defined and built with different types of defects. Moreover numerical simulations with a commercial finite elements code have been considered in addition.

\section{CARIDDI INTEGRAL FORMULATION}

The CARIDDI code is based on an integral formulation of the eddy current problem [1]. The integral formulation is obtained by assuming as unknown the electric vector potential $\mathbf{T}$ expanded in terms of edge elements based shape functions and applying the Galerkin method. Uniqueness is enforced by the tree-cotree decomposition [4]. The eddy current density is given by $\mathbf{J}=\nabla \times \mathbf{T}$ where $\mathbf{T}(\mathbf{r})=\sum_{k} I_{k} \mathbf{N}_{k}(\mathbf{r})$ and $\mathbf{N}_{k}$ is the $k$ th shape function. For time harmonic fields (the $\exp (j \omega t)$ time dependence is assumed), linear and nonmagnetic materials, the

Digital Object Identifier 10.1109/TMAG.2005.844357 column vector $\mathbf{I}$ of the degrees of freedom $I_{k}$ 's is solution of the linear system $\mathbf{Z I}=\mathbf{V}$ where $\mathbf{Z}=\mathbf{R}+j \omega \mathbf{L}, \mathbf{R}$, and $\mathbf{L}$ are properly defined matrices and $\mathbf{V}$ is a column vector depending on the assigned source current density. We highlight that only the conductive domain needs to be discretized, that regularity conditions at infinity are automatically taken into account and that $L$ is a full matrix whereas $R$ is a sparse matrix.

In the framework of ECT, two different lines have been pursued to reduce the computational cost and to increase the accuracy. The first takes advantage of the fact that in many practical situations: i) ECT is applied to image spatially localized conductivity perturbations (crack detection and imaging) and ii) it is relatively easy to locate a subregion $V_{T}$ (the tentative region) of the conductor that is candidate to contain the crack. This can be properly taken into account to obtain an integral formulation yielding a reduced linear system of the order of the shape functions $\mathbf{N}_{k}$ not vanishing in $V_{T}$. Specifically, (see [3], [4] for details) the unknown vector $\mathbf{I}$ can be decomposed as $\mathbf{I}=\mathbf{K x}+\mathbf{Q} \boldsymbol{\alpha}+\boldsymbol{\beta}_{0}$ where the column vector $\mathbf{x}$ accounts for the current density flowing outside $V_{T}, \boldsymbol{\alpha}$ accounts for the current density flowing in $V_{T}$ but outside the crack (assumed to be contained in $V_{T}$ ), $\mathbf{K}$ and $\mathbf{Q}$ are suitably defined matrices (see [5], [6]) and $\boldsymbol{\beta}_{0}$ is a vector arising from the superposition principle (the effect of the crack is obtained by adding a suitable imposed source current density in the crack volume). The effectiveness of the previous decomposition lies in the possibility of computing $\alpha$ by solving a "small" linear system and obtaining $\mathrm{x}$ from $\boldsymbol{\alpha}$ and $\boldsymbol{\beta}_{0}$ by linear mapping: $\mathbf{x}=\mathbf{A} \boldsymbol{\alpha}+\mathbf{C} \boldsymbol{\beta}_{0}$ where $\mathbf{A}$ and $\mathbf{B}$ are suitable matrices [5], [6].

The second line for efficiently computing the eddy currents within the ECT framework, consists of reducing the computational cost for the ZI matrix-vector product in order to solve $\mathbf{Z I}=\mathbf{V}$ by an iterative method such as GMRES. The computational cost for computing this product comes solely from the full matrix $\mathbf{L}$. Therefore, the key problem is the development of an efficient algorithm for computing the LI product.

To this purpose, it is worth noting that the $i$ th component of the $\mathbf{L I}$ product is given by $\int_{V_{t}} \mathbf{A} \cdot \nabla \times \mathbf{N}_{i} d V$ where $\mathbf{A}$ is the vector potential produced by the current density represented by 
the degrees of freedom of $\mathbf{I}$. Therefore, the central problem is the rapid computation of the vector potential produced by $\mathbf{I}$. To this purpose, we have exploited two different strategies, namely the precorrected FFT [7] and the FMM [8]. In both approaches we have exploited the feature whereby the far field produced by a given source current density does not depend on the details of the source distribution. Therefore, we treated the far and the near interactions separately. The near interactions can be accounted for by using a sparse matrix $\mathbf{L}^{\text {near}}$, whereas the far interactions by using a full matrix $\mathbf{L}^{\text {far }}$, i.e., $\mathbf{L}=\mathbf{L}^{\text {far }}+\mathbf{L}^{\text {near }}$. The precorrected FFT and the FMM differ in the method used for computing the far distance interactions (and, consequently, the near interactions).

In the precorrected FFT, we replace the current density described by $\mathbf{I}$ with a properly chosen (see [7] for details) source onto a regular Cartesian grid containing the conducting domain. Then, the vector potential produced by these sources is computed by means of the FFT thanks to the translationally invariant structure of the static free space Green function appearing in (1).

In the FMM, initially proposed in [9] for solving the Laplace equation and extended in [7], [8] for solving eddy current problems, the computation of the far interactions is carried out by using multipoles expansion. Specifically, the vector potential involved in the computation of the interactions with the $i$ th shape function is written as $\mathbf{A}=\mathbf{A}^{\text {near }}+\mathbf{A}^{\text {far }}$ where $\mathbf{A}^{\text {near }}(\mathbf{r})=\left(\mu_{0}\right) /(4 \pi) \int_{B_{i}}\left(\mathbf{J}\left(\mathbf{r}^{\prime}\right)\right) /\left(\left|\mathbf{r}-\mathbf{r}^{\prime}\right|\right) d V^{\prime}, \mathbf{A}^{\text {far }}(\mathbf{r})=$ $\left(\mu_{0}\right) /(4 \pi) \int_{V_{c} \backslash B_{i}}\left(\mathbf{J}\left(\mathbf{r}^{\prime}\right)\right) /\left(\left|\mathbf{r}-\mathbf{r}^{\prime}\right|\right) d V^{\prime}, B_{i}$ is a cubic box of size $l$ containing the support of $\nabla \times \mathbf{N}_{i}$ and $\mathbf{J}$ is the current density represented by $\mathbf{I}$. Each component of the vector potential $\mathbf{A}^{\text {far }}$ is harmonic in $B_{i}$ and it can, therefore, be expanded by a local expansion $\mathbf{A}^{\text {far }}(\mathbf{r})=$ $\left(\mu_{0}\right) /(4 \pi) \sum_{n=0}^{p} \sum_{m=-n}^{m} \mathbf{L}_{n}^{m} \boldsymbol{r}^{n} \boldsymbol{Y}_{n}^{m}(\theta, \phi)+O\left(\boldsymbol{l}^{-1} 3^{-(p+1) / 2}\right)$ where $\mathbf{L}_{n}^{m}$ is the vector of the local expansion coefficients and $p$ is the order of the local expansion. Consequently, $(\mathbf{L I})_{i}=\left(\mathbf{L}^{\text {near }} \mathbf{I}\right)_{i}+\left(\mu_{0}\right) /(4 \pi) \sum_{n=0}^{p} \sum_{m=-n}^{m} \mathbf{L}_{n}^{m} \mathbf{M}_{i, n}^{-m}+$ $O\left(\boldsymbol{l}^{-1} 3^{-(p+1) / 2}\right)$ where $\mathrm{M}_{i, n}^{-m}$ is the multipole of degree $n$ and order $m$ of $\nabla \times \mathbf{N}_{i}$ and $\mathbf{L}^{\text {near }}$ a sparse matrix. The efficiency of this scheme stems from the fact that the FMM allows the coefficients $\mathbf{L}_{n}^{m}$ to be recursively computed for any box, order and degree, with a nearly $O(n)$ computational cost.

\section{DiSCRETE GEOMETRIC FORMULATION}

We introduce in the domain of interest (containing the conductive region $V_{c}$ ) a pair of interlocked cell complexes: the primal $K$ and its dual $\tilde{K},[10],[11]$. The $p$-cells of $K=\{N, E, F, V\}$ are simplices, such as nodes, edges, faces (triangles) and volumes (tetrahedra) all endowed with inner orientation. The $p$-cells of $\tilde{K}=\{\tilde{N}, \tilde{E}, \tilde{F}, \tilde{V}\}$ are all endowed with outer orientation and are obtained from $K$ according to the barycentric subdivision. The pair $(K, \tilde{K})$ forms the mesh. The mutual interconnections between the cell complex $K, \tilde{K}$ are described by the incidence matrices: $\mathbf{G}$ between edges and nodes, $\mathbf{C}$ between faces and edges, and $\mathbf{D}$ between volumes and faces. The matrices $\tilde{\mathbf{G}}=\mathbf{D}^{T}, \tilde{\mathbf{C}}=\mathbf{C}^{T}$, and $\tilde{\mathbf{D}}=-\mathbf{G}^{T}$ describe the mutual interconnections of $\tilde{K}$. The arrays of Degree of Freedoms can be associated univocally to the elements of $K$ or $\tilde{K}$. We have that $\mathbf{U}$ is the array of voltages on primal edges, $\boldsymbol{\Phi}$ is the array of fluxes on primal faces, $\mathbf{F}$ is the array of magnetic voltages on dual edges and $\mathbf{I}$ is the array of currents on dual faces. In our linear eddy current problem with sinusoidal source currents, we apply the complex transformation to the above time harmonic arrays. The physical laws of the eddy-current problem, can be written exactly, regardless of the size of the particular mesh, as follows $\mathbf{D} \Phi=0$ (Gauss' law), $\tilde{\mathbf{C}} \mathbf{F}=\mathbf{I}$ (Ampère's law), $\mathbf{C} \mathbf{U}=-\boldsymbol{i} \omega \boldsymbol{\Phi}$ (Faraday's law), $\tilde{\mathbf{D I}}=0$ (continuity law).

Constitutive equations in discrete form are a finite dimensional linear operator (i.e., a matrix) linking some physical variables referred to a complex with other physical variables referred to the other complex. In our discrete quasistatic problem, the discrete magnetic and Ohm's constitutive equations are respectively $\mathbf{F}=\boldsymbol{\nu} \Phi, \mathbf{I}=\boldsymbol{\sigma} \mathbf{U}$ where $\boldsymbol{\nu}, \boldsymbol{\sigma}$ are two square matrices ( $F \times F$ and $E \times E$ respectively) containing material properties and metric notions. These matrices can be derived using a geometric approach [2], [12] under the assumption of uniformity (or uniformity in subregions) of the fields in each primal cell, while reluctivity and conductivity are assumed as constants within each tetrahedron.

One possible way to solve the discrete eddy-current problem is to introduce the array $\boldsymbol{\chi}$ of gauge functions values on primal nodes of the conducting region and the array a of circulations of the magnetic vector potential on primal edges. In this way Gauss' law and Faraday's law are satisfied identically when we write respectively $\boldsymbol{\Phi}=\mathbf{C}$ a and $\mathbf{U}=-\boldsymbol{i} \omega(\mathbf{a}+\mathbf{G} \chi)$, being $\mathbf{D C}=0$ and $\mathbf{C G}=0$.

A first set of equations, relative to the primal edges, is derived by substituting in Ampère's law the discrete magnetic constitutive equation, where the array of fluxes is expressed in terms of a. We obtain $\tilde{\mathbf{C}} \boldsymbol{\nu} \mathbf{C a}=\mathbf{I}$, where $\mathbf{I}$ is null in nonconducting regions. A second set of equations can be written, relative to the primal edges in $V_{c}$ by substituting in Ohm's constitutive equation, Faraday's law and the previous relation, obtaining $[\tilde{\mathbf{C}} \boldsymbol{\nu} \mathbf{C}+\boldsymbol{i} \omega \boldsymbol{\sigma}] \mathbf{a}+\boldsymbol{i} \omega \boldsymbol{\sigma} \mathbf{G} \boldsymbol{\chi}=\mathbf{0}$. The last set of equations is written for the primal nodes in $V_{c}$, by substituting the Ohm constitutive equation and Faraday's law in the continuity equation. We obtain $\boldsymbol{i} \omega \tilde{\mathbf{D}} \sigma \mathbf{a}+\boldsymbol{i} \omega \tilde{\mathbf{D}} \sigma \mathbf{G} \chi=\mathbf{0}$. It has been proven (see [11] for details) that $\tilde{\mathbf{C}} \boldsymbol{\nu} \mathbf{C}$ is symmetric positive semidefinite (and analogously also for $\tilde{\mathbf{D}} \sigma \mathbf{G}$ ). It coincides with the one that can be obtained using edge elements based on the Whitney complex. On the contrary the $\sigma$ matrix is not symmetric, which makes the difference with standard finite elements equations. A code based on the above described formulation has been implemented and optimized using FORTRAN 90 as the programming language and NAG library routines to iteratively solve the final linear system with conjugate gradient without gauging techniques.

We modeled the volumetric defect, assumed to be perfectly insulating, with a collection of dual faces. Then we imposed a zero current across each of the dual faces approximating the defect. Thanks to the linearity property, with a first eddy current analysis we computed the currents crossing the defect dual faces, when the defect volume is treated as a conductor. Then we performed a second eddy-current analysis, without the source current, feeding the set of defect dual faces with a current opposite to that in the flawless case. In order to solve this second problem, we modified the equation relative to edges and nodes 

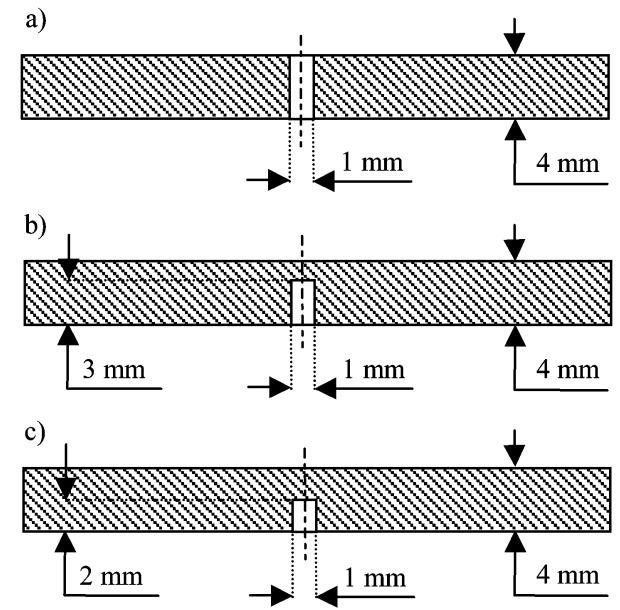

Fig. 1. Geometry of the benchmark problems (a), (b), (c).

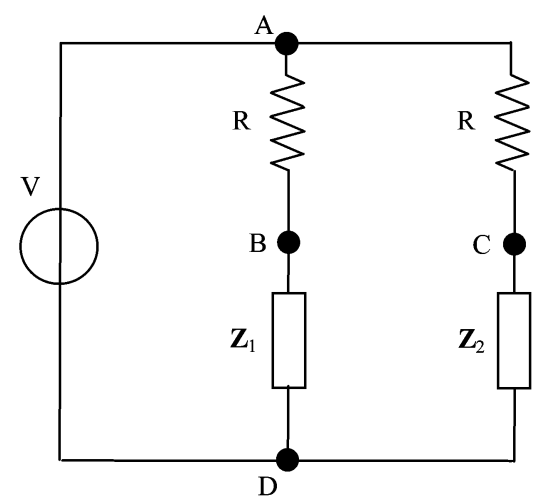

Fig. 2. Electrical circuit linked to probe coil.

of the conductor as described in [2]. In this way the impedance variation becomes the ratio between the induced voltage in the second eddy-current analysis and the impressed current per turn in the coil, used in the first analysis.

\section{BENCHMARKS AND EXPERIMENTAL SET-UP}

The numerical and experimental data were compared with respect to the three benchmarks a), b), and c) described in the following. On a $4 \mathrm{~mm}$ thick aluminum plate there is an artificial defect of cylindrical shape, $1 \mathrm{~mm}$ in diameter and 4, 3, and 2 $\mathrm{mm}$ in height, respectively (see Fig. 1). The detection technique based on the impedance variation of a probe coil is well known and widely studied and used for many applications. In particular it is possible to detect a thin crack in a metallic body by indirect measurement of the impedance variation of a probe coil, using the electrical circuit represented in Fig. 2. $V$ is an ac voltage source, $R$ is a bridge resistance, $Z_{1}$ is the impedance of a probe coil placed on a metallic plate, $Z_{2}$ is the impedance of a reference coil. The probe coil has 400 turns, is $10 \mathrm{~mm}$ in height, $12 \mathrm{~mm}$ in inner diameter, $18 \mathrm{~mm}$ in outer diameter and $0.5 \mathrm{~mm}$ in average lift-off. The reference coil is identical to the probe coil described above and is placed in a defect-free region of the metallic plate. The values $Z_{2}$ and $Z_{1}$ coincide when the probe coil is far from the defect location. It is possible to determine the defect location in the metallic plate by moving the probe coil on

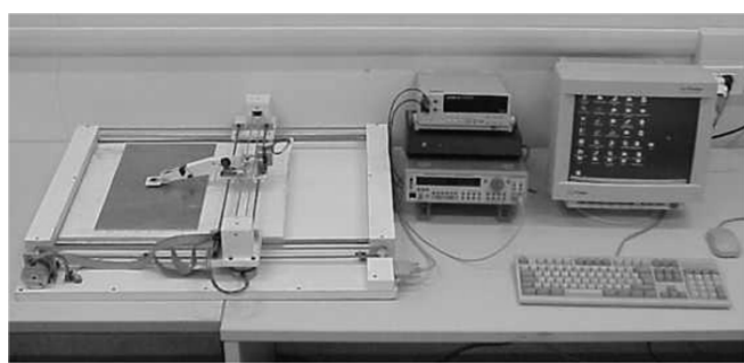

Fig. 3. Picture of experimental set-up.

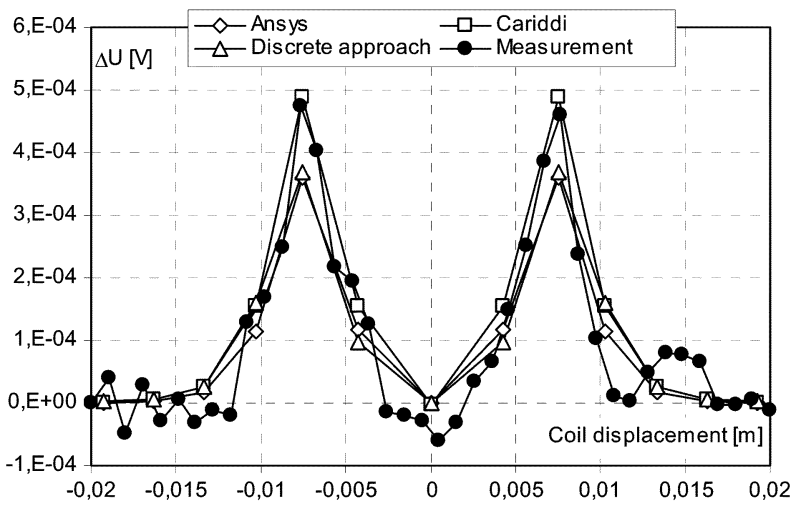

Fig. 4. Comparison of the three different numerical formulations with measurements (benchmark a).

the metallic plate surface and by measuring the voltage $\mathrm{V}_{\mathrm{BC}}$ between the nodes B and C (see Fig. 2). This particular topology of the electric circuit gives an output signal $\mathrm{V}_{\mathrm{BC}}$ which is immune with respect to the change of the voltage source value and to the thermal phenomena of the probe coil. Critical points for the experimental procedure are the positioning system for the probe coil with respect to the cracks on the metallic plate and the parameter acquisition system. The experimental set-up (see Fig. 3) consists of a two-dimensions automatic positioning system: 1 ) with a resolution of $0.1 \mathrm{~mm} ; 2$ ) a signal voltage analyzer with a resolution of $1 \mu \mathrm{V}$ for the $\mathrm{V}_{\mathrm{BC}}$ measurement; 3) an amplified signal generator for the electrical circuit supply; and 4) a dedicated software + digital control. In particular the probe coil path and the acquisition time of each point of measure can be computer-controlled [3].

\section{NUMERICAL RESULTS}

In order to compare the results we also used a commercial code (ANSYS) based on the differential formulation of magnetic vector potential $A$ and of the electric scalar potential V inside the conducting region [13], using 6194 hexahedral elements. With the discrete geometric formulation we used from 35000 (benchmark 1) to 45000 (benchmarks 2 and 3) tetrahedral (10546 elements in the conductor), taking 90 s of CPU time, as maximum, on a portable $\mathrm{PC}$ with $1 \mathrm{~GB}$ Ram at $1.8 \mathrm{GHz}$. The voltage $\Delta U\left(\mathrm{~V}_{\mathrm{BC}}\right.$ variation) from the integral, the discrete and the commercial FE formulations are compared with each other and with the experimental data, in Fig. 4 (benchmark a), in Fig. 5 (benchmark b) and in Fig. 6 (benchmark c). For the CARIDDI integral formulation we realized that a minimal mesh made of 4224 elements (discretizing the conducting region only) was 


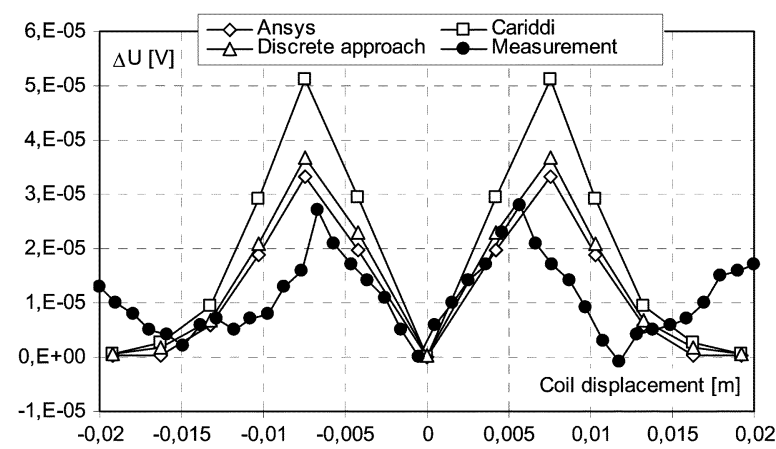

Fig. 5. Comparison of the three different numerical formulations with measurements (benchmark b).

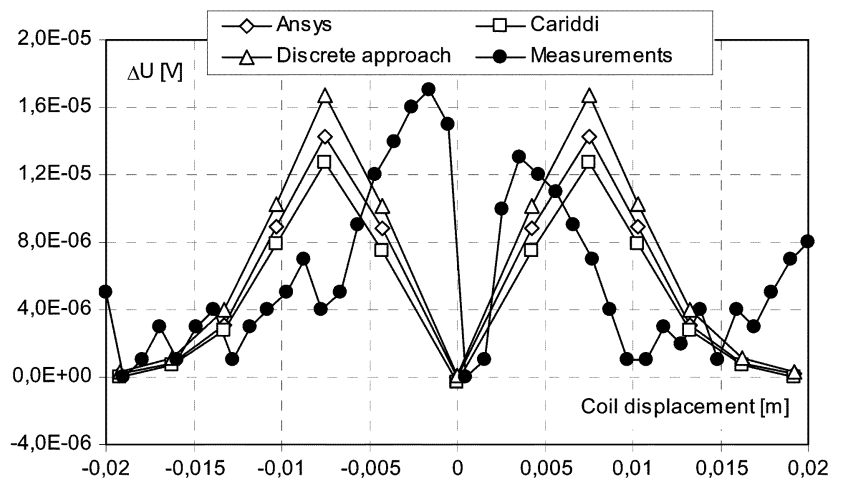

Fig. 6. Comparison of the three different numerical formulations with measurements (benchmark c).

enough to reach an accuracy within a few percent. This mesh involved 9378 unknowns, taking $164 \mathrm{~s}$ on a Beowulf parallel machine (sixteen nodes, each node consisting of a PIII- $450 \mathrm{MHz}$, 512 MB RAM, BGB HDD).

We have seen a general agreement among the computed data, like the ones reported in the paper, for all the benchmarks problems studied during the MADEND program.

This agreement is also extended to the measured data, if we take into account the noise of the signal due to lift-off and in- strument uncertainties at signal levels of a few microvolts, such as in our case.

\section{CONCLUSION}

This paper is a report of two numerical approaches studied and applied to nondestructive eddy current testing and evaluation during the MADEND Italian scientific research program. The examples of application to real eddy current evaluation benchmarks, and the general agreement either with the computed results, or with the measured data, suggest that the formulations and the numerical approaches presented in the paper can be considered as useful tools for the numerical modeling and design of eddy current diagnostics devices.

\section{REFERENCES}

[1] R. Albanese and G. Rubinacci, Proc. Inst. Elect. Eng., vol. 135, Sep. 1988, pp. 457-462.

[2] F. Trevisan, IEEE Trans. Magn., vol. 40, no. 2, pp. 1314-1317, Mar. 2004.

[3] E. Cardelli and A. Faba, Proc. XII Int. Symp. Theoretical Electrical Engineering (ISTET 2003), vol. 1, Warsaw, Poland, Jul. 6-9, 2003, p. 167.

[4] R. Albanese and G. Rubinacci, Adv. Imag. El. Phys., vol. 102, pp. 1-86, 1998.

[5] G. Rubinacci, A. Tamburrino, S. Ventre, and F. Villone, Int. J. Applied Electromagn. Mech., vol. 19, pp. 345-349, 2004.

[6] — E'NDE, Electromagnetic Non-Destructive Evaluation (VIII), T. Sollier et al., Eds. Amsterdam, The Netherlands: IOS Press, 2004, pp. 77-84.

[7] R. Albanese, G. Rubinacci, A. Tamburrino, S. Ventre, and F. Villone, COMPEL, vol. 20, no. 2, pp. 317-331, 2001.

[8] G. Rubinacci, A. Tamburrino, S. Ventre, and F. Villone, IEEE Trans. Magn., vol. 40, no. 2, pp. 1290-1293, Mar. 2004.

[9] L. Greengard and V. Rokhlin, J. Comput. Phys., vol. 73, pp. 325-348, 1987.

[10] E. Tonti, IEEE Trans. Magn., vol. 38, no. 2, pp. 333-336, Mar. 2002.

[11] A. Bossavit and L. Kettunen, IEEE Trans. Magn., vol. 36, no. 4, Jul. 2000.

[12] F. Trevisan and L. Kettunen, IEEE Trans. Magn., vol. 40, no. 2, pp. 361-365, Mar. 2004.

[13] O. Biro and K. Preis, IEEE Trans. Magn., vol. 25, no. 4, pp. 3145-3159, Jul. 1989.

Manuscript received June 6, 2004. 